\title{
Machine Learning with Semantic-Based Distances Between Sentences for Textual Entailment
}

\author{
Daniel Ferrés \\ TALP Research Center \\ Software Department \\ Universitat Politècnica de Catalunya \\ dferreselsi.upc . edu
}

\author{
Horacio Rodríguez \\ TALP Research Center \\ Software Department \\ Universitat Politècnica de Catalunya \\ horaciodlsi.upc.edu
}

\begin{abstract}
This paper describes our experiments on Textual Entailment in the context of the Third Pascal Recognising Textual Entailment (RTE-3) Evaluation Challenge. Our system uses a Machine Learning approach with Support Vector Machines and AdaBoost to deal with the RTE challenge. We perform a lexical, syntactic, and semantic analysis of the entailment pairs . From this information we compute a set of semanticbased distances between sentences. The results look promising specially for the QA entailment task.
\end{abstract}

\section{Introduction}

This paper describes our participation in the RTE3 challenge. It is our first attempt to RTE and we have taken profit of an analysis of the approaches followed in previous challenges (see (Dagan et al., 2005), and (Bar-Haim et al., 2006) for overviews of RTE-1 and RTE-2). Our approach, however, is based on a set of semantic-based distance measures between sentences used by our group in previous contests in Question Answering (TREC 2004, see (Ferrés et al., 2005), and CLEF 2004, see (Ferrés et al., 2004)) , and Automatic Summarization (DUC 2006, see (Fuentes et al., 2006)). Although the use of such measures (distance between question and sentences in passages candidates to contain the answer, distance between query and sentences candidates to be included in the summary, ...) is different for RTE task, our claim is that with some modifications the approach can be useful in this new scenario.
The organization of this paper is as follows. After this introduction we present in section 2 a description of the measures upon which our approach is built. Section 3 describes in detail our proposal. Results are discussed in section 4. Conclusions and further work is finally included in section 5 .

\section{System Description}

Our approach for computing distance measures between sentences is based on the degree of overlapping between the semantic content of the two sentences. Obtaining the semantic content implies a depth Linguistic Processing. Upon this semantic representation of the sentences several distance measures are computed. We next describe such issues.

\subsection{Linguistic Processing}

Linguistic Processing (LP) consists of a pipe of general purpose Natural Language (NL) processors that performs tokenization, morphologic tagging, lemmatization, Named Entities Recognition and Classification (NERC) with 4 basic classes (PERSON, LOCATION, ORGANIZATION, and OTHERS), syntactic parsing and semantic labelling, with WordNet synsets, Magnini's domain markers and EuroWordNet Top Concept Ontology labels. The Spear ${ }^{1}$ parser performs full parsing and robust detection of verbal predicate arguments. The syntactic constituent structure of each sentence (including the specification of the head of each constituent) and the relations among constituents (subject, direct and indirect object, modifiers) are obtained. As a result

\footnotetext{
${ }^{1}$ Spear. http://www.lsi.upc.edu/ surdeanu/ spear.html
} 
of the performance of these processors each sentence is enriched with a lexical and syntactic language dependent representations. A semantic language independent representation of the sentence (called environment) is obtained from these analyses (see (Ferrés et al., 2005) for details). The environment is a semantic network like representation built using a process to extract the semantic units (nodes) and the semantic relations (edges) that hold between the different tokens in the sentence. These units and relations belong to an ontology of about 100 semantic classes (as person, city, action, magnitude, etc.) and 25 relations (mostly binary) between them (e.g. time_of_event, actor_of_action, location_of_event, etc.). Both classes and relations are related by taxonomic links (see (Ferrés et al., 2005) for details) allowing inheritance. Consider, for instance, the sentence "Romano_Prodi 1 is 2 the 3 prime 4 minister 5 of 6 Italy 7". The following environment is built:

i_en_proper_person(1), entity_has_quality(2), entity(5), i_en_country(7), quality(4), which_entity(2,1), which_quality(2,5), $\bmod (5,7)$, $\bmod (5,4)$.

\subsection{Semantic-Based Distance Measures}

We transform each environment into a labelled directed graph representation with nodes assigned to positions in the sentence, labelled with the corresponding token, and edges to predicates (a dummy node, 0 , is used for representing unary predicates). Only unary (e.g. entity(5) in Figure 1) and binary (e.g. in Figure 2 which_quality $(2,5)$ ) predicates are used. Over this representation a rich variety of lexico-semantic proximity measures between sentences have been built. Each measure combines two components:

- A lexical component that considers the set of common tokens occurring in both sentences. The size of this set and the strength of the compatibility links between its members are used for defining the measure. A flexible way of measuring token-level compatibility has been set ranging from word-form identity, lemma identity, overlapping of WordNet synsets, approximate string matching between Named Entities etc. For instance, "Romano Prodi" is lex- ically compatible with "R. Prodi" with a score of 0.5 and with "Prodi" with a score of 0.41 . "Italy" and "Italian" are also compatible with score 0.7. This component defines a set of (partial) weighted mapping between the tokens of the two sentences that will be used as anchors in the next component.

- A semantic component computed over the subgraphs corresponding to the set of lexically compatible nodes (anchors). Four different measures have been defined:

- Strict overlapping of unary predicates.

- Strict overlapping of binary predicates.

- Loose overlapping of unary predicates.

- Loose overlapping of binary predicates.

The loose versions allow a relaxed matching of predicates by climbing up in the ontology of predicates (e.g. provided that $\mathrm{A}$ and $\mathrm{B}$ are lexically compatible, i_en_city $(A)$ can match i_en_proper_place $(B), i_{-} e n_{-} p r o p e r \_n a m e d \_e n t i t y(B)$, location $(B)$ or entity $(B))^{2}$. Obviously, loose overlapping implies a penalty on the score that depends on the length of the path between the two predicates and their informative content.

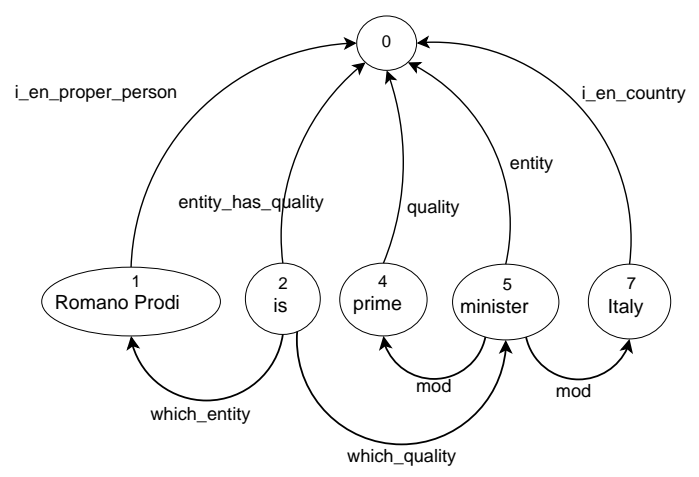

Figure 1: Example of an environment of a sentence.

${ }^{2}$ The ontology contains relations as i_en_city
isa i_en_proper_place, i_en_proper_place isa
$\begin{aligned} & \text { i_en_proper_named_entity, proper_place isa location, } \\ & \text { i_en_proper_named_entity isa entity, location isa entity }\end{aligned}$




\section{System Architecture}

We have adapted the set of measures described before for RTE in the following way:

1. We follow a Machine Learning (ML) approach for building a classifier to perform the RTE task. In previous applications the way of weighting and combining the different measures was based on a crude optimization using a development corpus.

2. We extract a more complex set of features for describing the semantic content of the Text $(\mathrm{T})$ and the Hypothesis $(\mathrm{H})$ as well as the set of semantic measures between them. Table 1 contains a brief summary of the features used.

3. We perform minor modifications on the tokenlevel compatibility measures for dealing with the asymmetry of the entailment relation (basically using the hyponymy and the verbal entailment relations of WordNet)

4. We add three new task-specific features (see Table 1)

The overall architecture of the system is depicted in Figure 2. As usual in ML, the system proceeds in two phases, learning and classification. The left side of the figure shows the learning process and the right part the classification process. The set of examples (tuples H, T) is first processed, in both phases, by LP for obtaining a semantic representation of the tuple $\left(H_{\text {sem }}\right.$ and $\left.T_{\text {sem }}\right)$. From this representation a Feature Extraction component extracts a set of features. This set is used in the learning phase for getting a classifier that is applied to the set of features of the test, during the classification phase, in order to obtain the answer.

\section{Experiments}

Before the submission we have performed a set of experiments in order to choose the Machine Learning algorithms and the training sets to apply in the final submission.

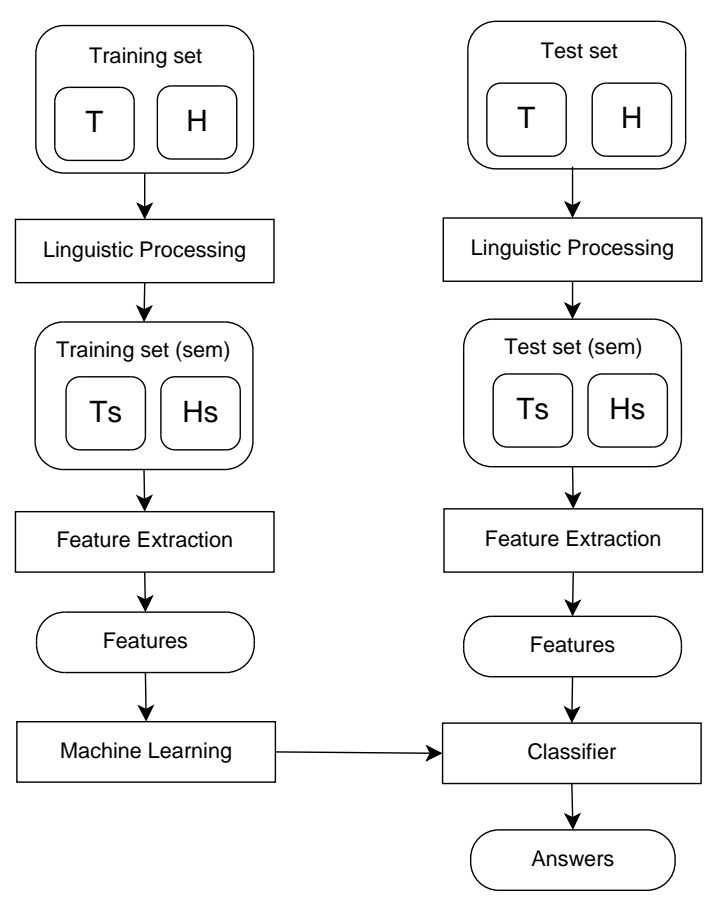

Figure 2: System Architecture.

\subsection{Machine Learning Experiments}

We used the WEKA $^{3}$ ML platform (Witten and Frank, 2005) to perform the experiments. We tested 9 different ML algorithms: AdaBoostM1, Bayes Networks, Logistic Regression, MultiBoostAB, Naive Bayes, RBF Network, LogitBoost (Simple Logistic in WEKA), Support Vector Machines (SMO in WEKA), and Voted Perceptron. We used the previous corpora of the RTE Challenge (RTE-1 and RTE2 ) and the RTE-3 development test. A filtering process has been applied removing pairs with more than two sentences in the text or hypothesis, resulting a total of 3335 Textual Entailment (TE) pairs. The results over 10-fold-Cross-Validation using a data set composed by RTE-1, RTE-2, and RTE-3 development set are shown in Table 2.

The results shown that AdaBoost, LogitBoost, and $S V M$ obtain the best results. Then we selected $A d$ aBoost and $S V M$ to perform the classification of the RTE-3 test set. The $S V M$ algorithm tries to compute the hyperplane that best separates the set of training examples (the hyperplane with maximum margin) (Vapnik, 1995). On the other hand, AdaBoost com-

\footnotetext{
${ }^{3}$ WEKA. http://www.cs.waikato.ac.nz/ml/ weka/
} 


\begin{tabular}{|l|c|l|}
\hline \multicolumn{1}{|c|}{ Features } & \#features & \multicolumn{1}{c|}{ Description } \\
\hline \hline semantic content of T & 12 & \#locations, \#persons, \#dates, \#actions, ... \\
\hline semantic content of H & 12 & $\ldots$ \\
\hline intersection of T and H & 12 & $\ldots$ \\
\hline Strict overlapping of unary predicates & 5 & $\begin{array}{l}\text { length of intersection } \\
\text { score of intersection } \\
\text { ratio of intersection related to shortest env } \\
\text { ratio of intersection related to longest env } \\
\text { ratio of intersection related to both (union of })\end{array}$ \\
\hline Strict overlapping of binary predicates & 5 & $\ldots$ \\
\hline Loose overlapping of unary predicates & 5 & $\ldots$ \\
\hline Loose overlapping of binary predicates & 5 & $\ldots$ \\
\hline Verbal entailment (WordNet) & 1 & V1 $\epsilon$ T, V2 $\epsilon$ H, such that V1 verbal_entails V2 \\
\hline Antonymy & 1 & $\begin{array}{l}\text { A1 } \epsilon \text { T, A2 } \epsilon \text { H, such that A1 and A2 are antonyms and } \\
\text { no token compatible with A2 }\end{array}$ \\
\hline \#occurs in H Negation & 1 & Difference between \# negation tokens in H and T \\
\hline
\end{tabular}

Table 1: Features used for classification with Machine Learning algorithms.

\begin{tabular}{|l||r|r|}
\hline Algorithm & \#correct & Accuracy \\
\hline \hline AdaBoostM1 & 1989 & 59.6402 \\
BayesNet & 1895 & 56.8216 \\
Logistic & 1951 & 58.5007 \\
MultiBoostAB & 1959 & 58.7406 \\
NaiveBayes & 1911 & 57.3013 \\
RBFNetwork & 1853 & 55.5622 \\
LogitBoost & 1972 & 59.1304 \\
SVM & 1972 & 59.1304 \\
VotedPerceptron & 1969 & 59.0405 \\
\hline
\end{tabular}

Table 2: Results over 10-fold-Cross-Validation using a filtered data set composed by RTE-1, RTE-2, and RTE-3 (a total of 3335 entailment pairs).

bines a set of weak classifiers into a strong one using lineal combination (Freund and Schapire, 1996). The idea is combining many moderately accurate rules into a highly accurate prediction rule. A weak learning algorithm is used to find the weak rules.

\subsection{Training Set Experiments}

We designed two experiments in order to decide the best training set to apply in the RTE-3 challenge. We performed an experiment using RTE-1 and RTE-2 data sets as a training set and the RTE-3 development set filtered (541 TE pairs) as a test set. In this experiment AdaBoost and SVM obtained accuracies of 0.6672 and 0.6396 respectively (see results in Table 3. We performed the same experiment joining the Answer Validation Exercise ${ }^{4}$ (AVE) 2006 English data set (Peñas et al., 2006) and the Microsoft Research Paraphrase Corpus ${ }^{5}$ (MSRPC) (Dolan et al., 2004) to the previous corpora (RTE-1 and RTE2) resulting a total of 8585 entailment pairs filtering pairs with a text or a hypothesis with more than 1 sentence. In our approach we considered that paraphrases were bidirectional entailments. The paraphrases of the MSRPC have been used as textual entailments in only one direction: the first sentence in the paraphrase has been considered the hypothesis and the second one has been considered the text.

Using the second corpus for training and the RTE3 development set as test set resulted in a notable degradation of accuracy (see Table 3).

\begin{tabular}{|l||r|r|}
\cline { 2 - 3 } \multicolumn{1}{c|}{} & \multicolumn{2}{c|}{ Accuracy } \\
\hline Algorithm & Corpus A & Corpus B \\
\hline \hline AdaBoost & $66.72 \%$ & $53.78 \%$ \\
SVM & $63.95 \%$ & $59.88 \%$ \\
\hline
\end{tabular}

Table 3: Results over the RTE-3 development set filtered (541 TE pairs) using as training set corpus A (RTE-1 and RTE-2) and corpus B (RTE-1, RTE-2, MSRPC, and AVE2006 English)

Finally, we performed a set of experiments to detect the contribution of the different features used for Machine Learning. These experiments revealed that

\footnotetext{
${ }^{4}$ AVE. http: //nlp. uned.es/QA/AVE

${ }^{5}$ MSRPC. http://research.microsoft.com/ $\mathrm{nlp} / \mathrm{ms}$ __paraphrase.htm
} 
the three most relevant features were: Strict overlapping of unary predicates, Semantic content of Hypothesis, and Loose overlapping of unary predicates.

\subsection{Official Results}

Our official results at RTE-3 Challenge are shown in Table 4. We submitted two experiments: the first one with AdaBoost (run1) and the second one with $S V M$ (run2). Training data set for final experiments were corpus: RTE-1 (development and test), RTE2 (development and test), and RTE-3 development. The test set was the RTE-3 test set without filtering the entailments (text or hypothesis) with more than one sentence. In this case we joined multiple sentences in a unique sentence that has been processed by the LP component.

We obtained accuracies of 0.6062 and 0.6150 . In the QA task we obtained the best per-task results with accuracies of 0.7450 and 0.7000 with AdaBoost and $S V M$ respectively.

\begin{tabular}{|l|r|r|}
\cline { 2 - 3 } \multicolumn{1}{c|}{} & \multicolumn{2}{c|}{ Accuracy } \\
\hline Task & $\begin{array}{r}\text { run1 } \\
\text { AdaBoost }\end{array}$ & $\begin{array}{r}\text { run2 } \\
\text { SVM }\end{array}$ \\
\hline \hline IE & 0.4350 & 0.4950 \\
IR & 0.6950 & 0.6800 \\
QA & 0.7450 & 0.7000 \\
SUM & 0.5500 & 0.5850 \\
\hline Overall & 0.6062 & 0.6150 \\
\hline
\end{tabular}

Table 4: RTE-3 official results.

\section{Conclusions and Further Work}

This paper describes our experiments on Textual Entailment in the context of the Third Pascal Recognising Textual Entailment (RTE-3) Evaluation Challenge. Our approach uses Machine Learning algorithms (SVM and AdaBoost) with semantic-based distance measures between sentences. Although further analysis of the results is in process, we observed that our official per-task results at RTE-3 show a different distribution compared with the global results of all system at RTE- 2 challenge. The RTE- 2 pertask analysis showed that most of the systems scored higher in accuracy in the multidocument summarization (SUM) task while in our system this measure is low. Our system at RTE-3 challenge scored higher in the QA and IR tasks with accuracies of 0.7450 and 0.6950 respectively in the first run.

\section{References}

Roy Bar-Haim, Ido Dagan, Bill Dolan, Lisa Ferro, Danilo Giampiccolo, Bernardo Magnini, and Idan Szpektor. 2006. The second pascal recognising textual entailment challenge. In Proceedings of the Second PASCAL Challenges Workshop on Recognising Textual Entailment.

Ido Dagan, Oren Glickman, and Bernardo Magnini. 2005. The pascal recognising textual entailment challenge. In Joaquin Quiñonero Candela, Ido Dagan, Bernardo Magnini, and Florence d'Alché Buc, editors, MLCW, volume 3944 of Lecture Notes in Computer Science, pages 177-190. Springer.

Bill Dolan, Chris Quirk, and Chris Brockett. 2004. Unsupervised construction of large paraphrase corpora: exploiting massively parallel news sources. In $C O L-$ ING '04: Proceedings of the 20th international conference on Computational Linguistics, page 350, Morristown, NJ, USA. Association for Computational Linguistics.

Daniel Ferrés, Samir Kanaan, Alicia Ageno, Edgar González, Horacio Rodríguez, Mihai Surdeanu, and Jordi Turmo. 2004. The TALP-QA System for Spanish at CLEF 2004: Structural and Hierarchical Relaxing of Semantic Constraints. In Carol Peters, Paul Clough, Julio Gonzalo, Gareth J. F. Jones, Michael Kluck, and Bernardo Magnini, editors, CLEF, volume 3491 of Lecture Notes in Computer Science, pages 557-568. Springer.

Daniel Ferrés, Samir Kanaan, Edgar González, Alicia Ageno, Horacio Rodríguez, Mihai Surdeanu, and Jordi Turmo. 2005. TALP-QA System at TREC 2004: Structural and Hierarchical Relaxation Over Semantic Constraints. In Proceedings of the Text Retrieval Conference (TREC-2004).

Yoav Freund and Robert E. Schapire. 1996. Experiments with a new boosting algorithm. In International Conference on Machine Learning, pages 148-156.

Maria Fuentes, Horacio Rodríguez, Jordi Turmo, and Daniel Ferrés. 2006. Femsum at duc 2006: Semanticbased approach integrated in a flexible eclectic multitask summarizer architecture. In Proceedings of the Document Understanding Conference 2006 (DUC 2006). HLT-NAACL 2006 Workshop., New York City, NY, USA, June.

Anselmo Peñas, Álvaro Rodrigo, Valentín Sama, and Felisa Verdejo. 2006. Overview of the answer validation exercise 2006. In Working Notes for the 
CLEF 2006 Workshop. ISBN: 2-912335-23-x, Alicante, Spain, September.

Vladimir N. Vapnik. 1995. The nature of statistical learning theory. Springer-Verlag New York, Inc., New York, NY, USA.

Ian H. Witten and Eibe Frank. 2005. Data Mining: Practical Machine Learning Tools and Techniques, Second Edition (Morgan Kaufmann Series in Data Management Systems). Morgan Kaufmann, June.

\section{Acknowledgments}

This work has been supported by the Spanish Research Dept. (TEXT-MESS, TIN2006-15265-C0605). Daniel Ferrés is supported by a UPC-Recerca grant from Universitat Politècnica de Catalunya (UPC). TALP Research Center is recognized as a Quality Research Group (2001 SGR 00254) by DURSI, the Research Department of the Catalan Government. 